\title{
Holey metal films: From extraordinary transmission to negative-index behavior
}

\author{
A. Mary, ${ }^{1}$ Sergio G. Rodrigo, ${ }^{2}$ L. Martín-Moreno, ${ }^{2}$ and F. J. García-Vidal ${ }^{1}$ \\ ${ }^{1}$ Departamento de Física Teórica de la Materia Condensada, Universidad Autónoma de Madrid, E-28049 Madrid, Spain \\ ${ }^{2}$ Instituto de Ciencia de Materiales de Aragón (ICMA) and Departamento de Física de la Materia Condensada, \\ CSIC-Universidad de Zaragoza, E-50009 Zaragoza, Spain
}

(Received 30 July 2009; revised manuscript received 4 October 2009; published 29 October 2009)

\begin{abstract}
In this paper we study the common physical background of the phenomenon of extraordinary optical transmission in holey metal films and the emergence of the negative refractive index behavior in double fishnet structures. Here we provide further evidence that the resonant magnetic response of the latter structures is associated with the excitation of gap surface plasmon modes. The evolution of the optical response with the number of double fishnet layers is also addressed, finding that the effective refractive index reaches a converged value for a moderate number of layers.
\end{abstract}

DOI: 10.1103/PhysRevB.80.165431

PACS number(s): 78.20.Ci, 42.25.Bs, 73.20.Mf

\section{INTRODUCTION}

The phenomenon of extraordinary optical transmission (EOT) through two-dimensional arrays of holes (2DHA) discovered by Ebbesen et al. ${ }^{1}$ has generated great interest due to its fundamental importance for manipulating light at a subwavelength scale. ${ }^{2,3}$ From the beginning, it was realized that the spectral locations of the transmission resonances are related to those of the surface-plasmon polaritons (SPPs). ${ }^{4}$ This link between EOT and SPPs has been corroborated by subsequent theoretical works ${ }^{5}$ and it is now widely accepted that the resonant excitation of surface electromagnetic (EM) modes is the general mechanism behind the EOT phenomenon appearing both in the optical regime and at lower frequencies.

Recently, Zhang et al. ${ }^{6,7}$ have proposed and demonstrated a negative-index metamaterial (NIM) working at nearinfrared frequencies with a design very similar to that of a 2DHA. This NIM metamaterial is composed by a 2DHA penetrating completely into a metal-dielectric-metal film stack and is usually termed as double-fishnet (DF) structure. DF structures have received a lot of attention and the emergence of DF-based negative refractive index (NRI) at visible, ${ }^{8}$ near infrared, ${ }^{9,10}$ and also at microwave frequencies has been demonstrated. ${ }^{11-13}$ More recently, NIMs made of cascaded DF (CDF) structures were theoretically ${ }^{14,15}$ and experimentally ${ }^{16,17}$ investigated.

The aim of this paper is twofold: first, we establish a link between the physics underlying both EOT and NRI phenomena observed in 2DHA and DF structures. Here we give a detailed account of this relation that was previously reported in Ref. 18 and has been experimentally verified in the $\mathrm{GHz}$ range of the EM spectrum. ${ }^{19}$ Second, we study the evolution of the NRI response when going from a single DF structure to a multilayered CDF. The paper is organized as follows: in Sec. II we describe the quasianalytical theoretical formalism (coupled-mode method, CMM) that is used throughout this paper. By using this method, optical transmission and effective optical parameters can be obtained for both 2DHA structures (Sec. III) and DF systems (Sec. IV). Results obtained within the perfect electric conductor (PEC) approximation are compared with calculations done for a real metal $(\mathrm{Ag})$ at optical frequencies in which the numerical results are tested with those obtained from a virtually exact finite-difference time-domain (FDTD) method. In Sec. VI we also discuss the effect of the dielectric function and of the thickness of the dielectric slab on the negative-index behavior of a DF structure. The evolution from a single DF to a CDF is analyzed in detail in Sec. V. Finally, a summary of the main theoretical findings is given in Sec. VI.

\section{THEORETICAL FORMALISM}

Figure 1 illustrates the DF structure analyzed throughout this paper. As explained above, the DF structure consists of
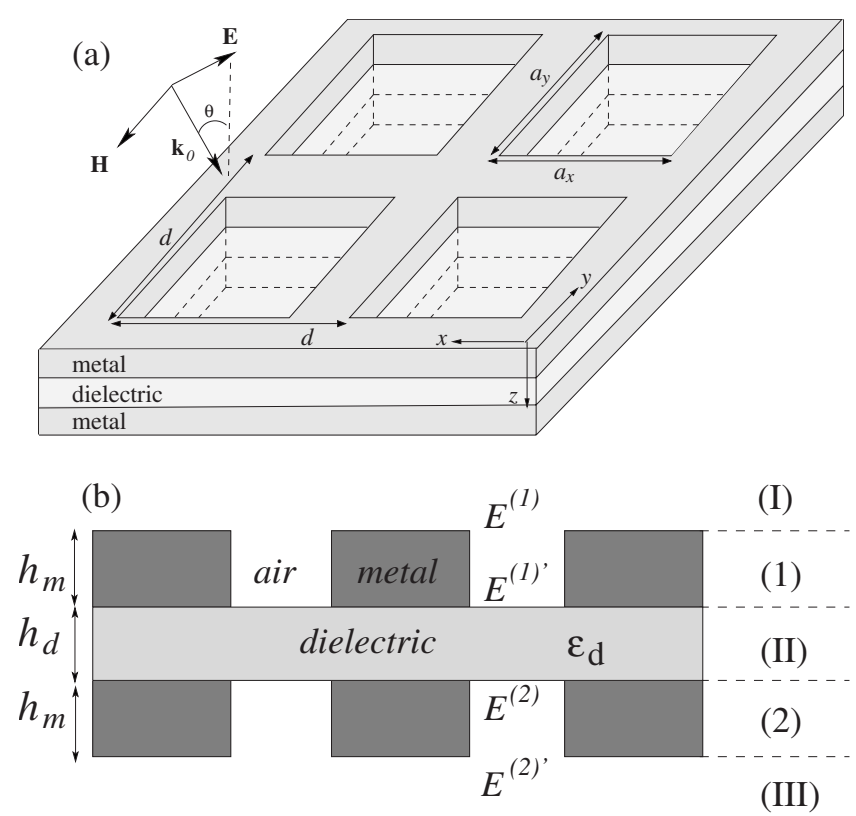

FIG. 1. (a) Schematic pictures of a DF structure: a square array of rectangular holes of side $a_{x}$ and $a_{y}$ perforated on two metallic films of thickness $h_{m}$. The perforated films are separated by a dielectric medium of thickness $h_{d}$ characterized by a dielectric constant $\epsilon_{d}$. Parameter $d$ defines the period of the array. (b) Schematic representation of the notation used for the modal amplitudes related to the DF structure. 
an infinite square array (period $d$ ) of rectangular holes of sides $a_{x}$ and $a_{y}$, perforated on two metallic slabs of thickness $h_{m}$ separated by a dielectric layer of thickness $h_{d}$. The 2DHA structure is obtained as a particular case of a DF by considering the limit $h_{d}=0$. In this case the thickness of the resulting single metallic layer would be $h=2 h_{m}$. For both structures (2DHA and DF), we are interested in looking at the transmission and reflection properties of this structure under illumination by a $p$-polarized plane wave.

Here in this section we present the main ingredients of the general theoretical framework used in this paper (a coupledmode method) whose application to DF structures was first reported in Ref. 20. This framework is based on the modal expansion of the EM fields in the different regions of the structure $^{21}$ assuming first that the metal behaves as a PEC. In this way, in reflection (I) and transmission (III) air regions the EM fields are expressed in terms of $s$ - and $p$-polarized plane waves whereas in the perforated metal films (regions 1 and 2) the EM fields are expanded by means of the TE and TM waveguide modes inside the holes. One of the main advantages of the coupled-mode method is that for holes with simple cross sections as rectangular or circular, the spatial dependence of the electric and magnetic fields associated with the different waveguide modes are known analytically. Region II corresponds to the dielectric slab located between the two metal films. As a difference with the experimental samples, in our study and in order to simplify the numerical calculations, we will assume that this dielectric layer is uniform (no holes), allowing an expansion of the EM fields in this region also in terms of plane waves. We will show later that similar results are obtained for perforated and nonperforated dielectric layers. The second step within the coupledmode method is to match the parallel components of the EM fields at the four interfaces forming the DF structure. The finite dielectric function of a real metal at optical frequencies can be incorporated into CMM by using surface-impedance boundary conditions on the horizontal metal-dielectric boundaries. The surface impedance of the metal, $Z_{s}$, is related to the frequency-dependent dielectric function of the metal, $\epsilon(\omega): Z_{s}=1 / \sqrt{\epsilon(\omega)}$. After the matching procedure, a system of linear equations for the expansion coefficients of the EM fields inside the holes $\left\{E_{\alpha}^{(n)}, E_{\alpha}^{(n)^{\prime}}\right\}$ is obtained

$$
\begin{gathered}
\left(G_{\alpha \alpha}-\Sigma_{\alpha}\right) E_{\alpha}^{(1)}+\sum_{\beta \neq \alpha} G_{\alpha \beta} E_{\beta}^{(1)}-G_{\alpha}^{V} E_{\alpha}^{(1)^{\prime}}=I_{\alpha}, \\
G_{\alpha}^{V} E_{\alpha}^{(1)}+\left(Q_{\alpha \alpha}+\Sigma_{\alpha}\right) E_{\alpha}^{(1)^{\prime}}+P_{\alpha \alpha} E_{\alpha}^{(2)} \\
+\sum_{\beta \neq \alpha}\left[Q_{\alpha \beta} E_{\beta}^{(1)^{\prime}}+P_{\alpha \beta} E_{\beta}^{(2)}\right]=0, \\
P_{\alpha \alpha} E_{\alpha}^{(1)^{\prime}}+\left(Q_{\alpha \alpha}+\Sigma_{\alpha}\right) E_{\alpha}^{(2)}+G_{\alpha}^{V} E_{\alpha}^{(2)^{\prime}} \\
+\sum_{\beta \neq \alpha}\left[P_{\alpha \beta} E_{\beta}^{(1)^{\prime}}+Q_{\alpha \beta} E_{\beta}^{(2)}\right]=0,
\end{gathered}
$$

$$
\left(G_{\alpha \alpha}-\Sigma_{\alpha}\right) E_{\alpha}^{(2)^{\prime}}+\sum_{\beta \neq \alpha} G_{\alpha \beta} E_{\beta}^{(2)^{\prime}}-G_{\alpha}^{V} E_{\alpha}^{(2)}=0,
$$

where $\alpha$ (and $\beta$ ) runs over the waveguide modes inside the holes. In this system of linear equations, the unknown $E_{\alpha}^{(n)}$ is related to the modal amplitude of mode $\alpha$ inside the holes, evaluated at the entrance surface of the $n$th metal layer ( $n$ is 1 or 2 depending on the metal layer considered). On the other hand, the primed expansion coefficient $\left(E_{\alpha}^{(n)^{\prime}}\right)$ accounts for the modal amplitude at the exit surface of the $n$th layer (see Fig. 1).

The different terms of this set of equations have a simple physical interpretation. $I_{\alpha}$ is the illumination term and basically measures the overlap between the incident plane wave (characterized by a parallel momentum $\mathbf{k}_{\mathbf{i}}$ and polarization $\sigma_{0}$ ) and the mode $\alpha$ of the hole array in the first metal layer $(n=1)$,

$$
I_{\alpha}=2 i \frac{Y_{\mathbf{k}_{\mathbf{i}} \sigma_{0}} S_{\alpha, \mathbf{k}_{\mathbf{i}} \sigma_{0}}}{1+Z_{s} Y_{\mathbf{k}_{\mathbf{i}} \sigma_{0}}},
$$

where $S_{\alpha, \mathbf{k}_{\mathbf{i}} \sigma_{0}}$ is the overlap integral and $Y_{\mathbf{k}_{\mathbf{i}} \sigma_{0}}$ is the admittance of the incident plane wave $\left(Y_{\mathbf{k}_{\mathbf{i}} s}=k_{z}^{i} / k_{0}\right.$ for the case of a $s$-polarized wave or $Y_{\mathbf{k}_{\mathbf{i}} p}=k_{0} / k_{z}^{i}$ for $p$ polarization). The $z$ component of the wave vector is $k_{z}^{i}=\sqrt{k_{0}^{2}-\left|k_{i}\right|^{2}}, k_{0}$ being the wave number of the incident plane wave, $k_{0}=2 \pi / \lambda$.

The EM coupling between the holes forming the $2 \mathrm{D}$ array is mediated by the term $G_{\alpha \beta}$ which can be expressed as a sum over diffracted plane waves in regions I or III. These plane waves are characterized by a parallel momentum $\mathbf{k}$ and polarization $\sigma$.

$$
G_{\alpha \beta}=i \sum_{\mathbf{k}, \sigma} \frac{Y_{\mathbf{k} \sigma} S_{\alpha, \mathbf{k} \sigma} S_{\beta, \mathbf{k} \sigma}^{*}}{1+Z_{S} Y_{\mathbf{k} \sigma}}
$$

where $S_{\mathbf{k} \sigma, \alpha}$ is the overlap integral between plane wave $\mathbf{k} \sigma$ and mode $\alpha{ }^{22}$ According to Bloch's theorem, $\mathbf{k}=\mathbf{k}_{\mathbf{i}}+\mathbf{G}, \mathbf{G}$ being a reciprocal-lattice vector.

The term $G_{\alpha}^{V}$ controls the coupling between the input and the output sides of the holes within a perforated metal layer

$$
G_{\alpha}^{V}=\frac{2 i Y_{\alpha} e^{i q_{z \alpha} h_{m}}}{e^{2 i q_{z \alpha} h_{m}}\left(1+Z_{S} Y_{\alpha}\right)^{2}-\left(1-Z_{S} Y_{\alpha}\right)^{2}},
$$

where the admittance of mode $\alpha, Y_{\alpha}$, is simply related to its propagation constant, $q_{z \alpha}: Y_{\alpha}=q_{z \alpha} / k_{0}$ for a TE mode while for a TM mode $Y_{\alpha}=k_{0} / q_{z \alpha}$.

The bouncing back and forth of the EM fields inside the holes is accounted for the term $\Sigma_{\alpha}$,

$$
\Sigma_{\alpha}=i Y_{\alpha} \frac{e^{2 i q_{z \alpha} h_{m}}\left(1+Z_{s} Y_{\alpha}\right)+\left(1-Z_{s} Y_{\alpha}\right)}{e^{2 i q_{z \alpha} h_{m}}\left(1+Z_{s} Y_{\alpha}\right)^{2}-\left(1-Z_{s} Y_{\alpha}\right)^{2}} .
$$

As we will show below, the resonant magnetic response of the DF structure is link to the EM coupling between the two perforated metal layers, $Q_{\alpha \beta}$ and $P_{\alpha \beta}$, whose analytical expressions are 


$$
Q_{\alpha \beta}=i \sum_{\mathbf{k}, \sigma} Y_{\mathbf{k} \sigma}^{d} S_{\alpha, \mathbf{k} \sigma} S_{\beta, \mathbf{k} \sigma}^{*} \frac{e^{2 i k_{z}^{d} h_{d}}\left(1+Z_{S} Y_{\mathbf{k} \sigma}^{d}\right)+\left(1-Z_{s} Y_{\mathbf{k} \sigma}^{d}\right)}{e^{2 i k_{z}^{d} h_{d}}\left(1+Z_{S} Y_{\mathbf{k} \sigma}^{d}\right)^{2}-\left(1-Z_{s} Y_{\mathbf{k} \sigma}^{d}\right)^{2}},
$$

$$
P_{\alpha \beta}=\sum_{\mathbf{k}, \sigma} 2 i Y_{\mathbf{k} \sigma}^{d} S_{\alpha, \mathbf{k} \sigma} S_{\beta, \mathbf{k} \sigma}^{*} \frac{e^{i k_{z}^{d} h_{d}}}{e^{2 i k_{z}^{d} h_{d}\left(1+Z_{S} Y_{\mathbf{k} \sigma}^{d}\right)^{2}-\left(1-Z_{S} Y_{\mathbf{k} \sigma}^{d}\right)^{2}},}
$$

where $k_{z}^{d}=\sqrt{\epsilon_{d} k_{0}^{2}-|k|^{2}}$ and the admittances of the plane waves inside the dielectric layer are $Y_{\mathbf{k s}}^{d}=k_{z}^{d} / k_{0}$ for a $s$-polarized wave and $Y_{\mathbf{k} p}^{d}=\epsilon_{d} k_{0} / k_{z}^{d}$ for $p$ polarization.

Once the different modal amplitudes are obtained, the EM fields in all regions forming the structure can be calculated and the reflection (region I) and transmission (region III) coefficients can be calculated by means of

$$
\begin{gathered}
r_{\mathbf{k}, \sigma}=\frac{1}{1+Z_{S} Y_{\mathbf{k} \sigma}} \sum_{\alpha} E_{\alpha}^{(1)} S_{\alpha, \mathbf{k} \sigma}-\frac{1-Z_{S} Y_{\mathbf{k} \sigma}}{1+Z_{S} Y_{\mathbf{k} \sigma}} \delta_{\mathbf{k} \sigma, \mathbf{k}_{\mathbf{i}} \sigma_{0}}, \\
t_{\mathbf{k}, \sigma}=-\frac{1}{1+Z_{S} Y_{\mathbf{k} \sigma}} \sum_{\alpha} E_{\alpha}^{(2)^{\prime}} S_{\alpha, \mathbf{k} \sigma} .
\end{gathered}
$$

Finally, the effective optical parameters (electric permittivity, $\epsilon_{e f f}$, magnetic permeability, $\mu_{e f f}$, and refractive index, $\left.n_{e f f}\right)$ are retrieved from the zero-order transmission and reflection coefficients by using the method based on $S$ parameters. ${ }^{23,24}$

The coupled-mode formalism can be extended straightforwardly to analyzing a CDF formed by a large number of metallic films, as it is shown in Sec. V. Conversely, the system of Eq. (1) can be easily reduced for the case of a single 2DHA. For this particular case, by imposing $h_{d}=0$ and $E_{\alpha}^{(1)^{\prime}}=-E_{\alpha}^{(2)}$, the system of linear equations transforms into

$$
\begin{aligned}
& \left(G_{\alpha \alpha}-\Sigma_{\alpha}\right) E_{\alpha}^{(1)}+\sum_{\beta \neq \alpha} G_{\alpha \beta} E_{\beta}^{(1)}-G_{\alpha}^{V} E_{\alpha}^{(2)^{\prime}}=I_{\alpha}, \\
& \left(G_{\alpha \alpha}-\Sigma_{\alpha}\right) E_{\alpha}^{(2)^{\prime}}+\sum_{\beta \neq \alpha} G_{\alpha \beta} E_{\beta}^{(2)^{\prime}}-G_{\alpha}^{V} E_{\alpha}^{(1)}=0,
\end{aligned}
$$

where the expressions for $G_{\alpha}^{V}$ and $\Sigma_{\alpha}$ are those of Eqs. (4) and (5) but $h_{m}$ must be replaced by $2 h_{m}$. Notice that the system of Eq. (10) is formally equivalent to that found in Ref. 25 for a 2DHA.

When dealing with subwavelength holes, a very good approximation to the transmission and reflection properties of a holey metal film can be achieved by considering only the fundamental mode $(\alpha \equiv 0)$ inside the holes which corresponds to the least decaying evanescent mode (the $\mathrm{TE}_{01}$ mode for the chosen polarization of the incident plane wave). In this way, the system of linear Eq. (1) reduces to a set of just four linear equations

$$
\begin{gathered}
(G-\Sigma) E^{(1)}-G_{0}^{V} E^{(1)^{\prime}}=I, \\
G^{V} E^{(1)}+(Q+\Sigma) E^{(1)^{\prime}}+P E^{(2)}=0,
\end{gathered}
$$

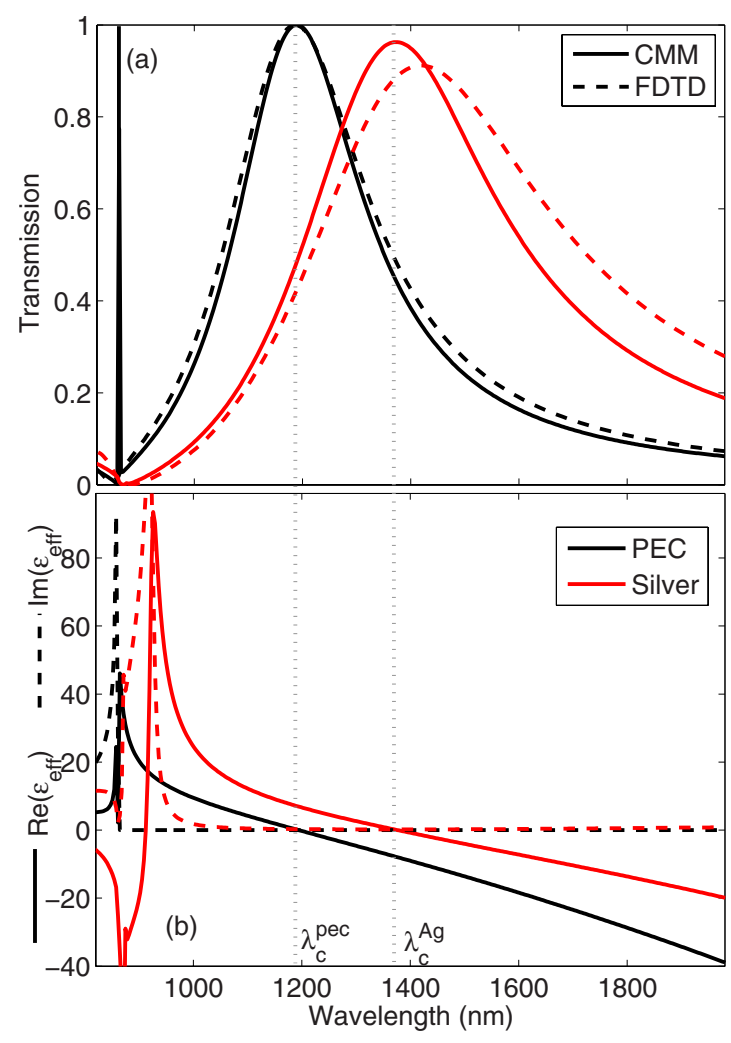

FIG. 2. (Color online) (a) Normal-incidence transmission versus wavelength for a 2DHA perforated on a PEC (black lines) and silver (red lines) film of thickness $h=60 \mathrm{~nm}$ and the period of the array is $d=860 \mathrm{~nm}$. The sides of holes are $a_{x}=295 \mathrm{~nm}$ and $a_{y}$ $=595 \mathrm{~nm}$. Solid curves: CMM calculations. Dashed curves: FDTD calculations. (b) Corresponding real (solid lines) and imaginary (dashed lines) of the effective permittivity $\epsilon_{\text {eff }}$ calculated by CMM versus wavelength. Dotted lines represent the position of the cutoff wavelength related to the fundamental TE mode $\left(\mathrm{TE}_{01}\right)$ when the metal is treated as a perfect conductor $\left(\lambda_{c}^{p e c}\right)$ and silver $\left(\lambda_{c}^{\mathrm{Ag}}\right)$.

$$
\begin{gathered}
P E^{(1)^{\prime}}+(Q+\Sigma) E^{(2)}+G^{V} E^{(2)^{\prime}}=0, \\
(G-\Sigma) E^{(2)^{\prime}}-G^{V} E^{(2)}=0,
\end{gathered}
$$

where the different terms $\left(G, \Sigma, G^{V}, Q\right.$, and $\left.P\right)$ have the same expressions as before but evaluated for the fundamental $\mathrm{TE}_{01}$ mode.

\section{EOT IN TWO-DIMENSIONAL HOLE ARRAYS}

First, let us briefly revisit the physical origin of the EOT phenomenon in the case of a 2DHA structure. Figure 2(a) renders the normal-incidence transmission spectra corresponding to a single metal layer perforated with a 2DHA (single fishnet structure). Solid curves have been obtained by using the coupled-mode method described above for PEC and Ag metal films by considering $h_{d}=0$. Dashed curves depict the corresponding transmission spectra as calculated with the FDTD method. It is clear that our quasianalytical formalism is able to capture accurately the main features observed in the FDTD spectra. The spectral locations and the 
corresponding linewidths of the several peaks are well reproduced by the simplified CMM calculations. For a silver film, the heights of the transmission peaks are higher and blueshifted in CMM with respect to FDTD results and is mainly due to the fact that, as a difference of FDTD, absorption within the vertical walls of the holes is neglected within CMM.

In the frequency range shown in the figure, there are two EOT peaks showing $100 \%$ transmittance in the PEC case. The first peak, that appears close to $\lambda=d$, is associated with the excitation of a surface EM mode. As the metal behaves as a PEC, this mode is not a real SPP but a geometrically induced SPP. ${ }^{26}$ The second peak emerges at around the cutoff wavelength of the fundamental TE mode $\lambda_{c}^{p e c}=1190 \mathrm{~nm}$. At this frequency, the propagation constant of the fundamental mode inside the holes is zero, which corresponds the usual Fabry-Pérot resonance condition. ${ }^{27}$ When the metal is treated as a real metal, due to the penetration of the EM fields inside the metal and the different effective propagation characteristics, the cutoff wavelength, $\lambda_{c}^{\mathrm{Ag}}$, shifts to longer wavelength with respect to the PEC case. ${ }^{28}$ Accordingly, the Fabry-Pérot resonance condition (and the corresponding transmission peak) is shifted to longer wavelengths. ${ }^{29}$

In Fig. 2(b) we study the evolution of the corresponding effective dielectric permittivity $\left(\boldsymbol{\epsilon}_{e f f}\right)$ of a 2DHA. This evolution can be understood by looking at the effective medium limit $(\lambda \gg d)$ where diffraction effects can be safely neglected. Inside the holes, the fundamental TE mode with propagation constant $q_{z}=\sqrt{k_{0}^{2}-\pi^{2} / a_{y}^{2}}$ will dominate the transmission process. Consequently, the system of Eq. (10) can be further simplified and the zero-order transmission coefficient ( $t$ ) can be written as

$$
t=-\frac{S G^{V} I}{(G-\Sigma)^{2}-\left(G^{V}\right)^{2}},
$$

where the overlap integral between the normally incident plane wave and the fundamental mode is $S=2 \sqrt{2 a_{x} a_{y}} /(d \pi)$. By using the analytical expressions for $G^{V}, G$, and $\Sigma$ in the PEC limit $\left(Z_{s}=0\right)$, we end up with an expression for $t$,

$$
t=\frac{4 Y_{0} Y|S|^{2} e^{i q_{z} h}}{\left[Y_{0}+Y|S|^{2}\right]^{2}-\left[Y_{0}-Y|S|^{2}\right]^{2} e^{2 i q_{z} h}},
$$

where $Y_{0}$ is the admittance of the $\mathrm{TE}_{01}$ mode, $Y_{0}=q_{z} / k_{0}$, and $Y$ is the admittance of the incident $p$-polarized plane wave, $Y=k_{0} / k_{z}^{i}$.

In order to extract the effective optical parameters of a 2DHA we need to compare first the results of Eq. (13) with the transmission coefficient for a $p$-polarized plane wave through a homogeneous film characterized by $\epsilon_{\text {eff }}$ and $\mu_{\text {eff }}$,

$$
t=\frac{4 \epsilon_{e f f} k_{z}^{\prime} k_{z}^{i} e^{i k_{z}^{\prime} h}}{\left(\epsilon_{e f f} k_{z}^{i}+k_{z}^{\prime}\right)^{2}-\left(\epsilon_{e f f} k_{z}^{i}-k_{z}^{\prime}\right)^{2} e^{2 i k_{z}^{\prime} h},}
$$

where $k_{z}^{\prime}$ is the $z$ component of the wave vector inside the effective medium. By comparing Eq. (14) with Eq. (13), an expression for $\epsilon_{e f f}$ can be extracted,

$$
\epsilon_{e f f}=\frac{1}{S^{2}}\left(1-\frac{\omega_{p}^{2}}{\omega^{2}}\right),
$$

where $\omega_{p}=\pi c / a_{y}$ is the cutoff frequency of the hole waveguide. As the $z$ component of the wave vector inside the effective medium $k_{z}^{\prime}=k_{0} \sqrt{\epsilon_{\text {eff }} \mu_{\text {eff }}}$ must be equal to the propagation constant of the fundamental mode, $q_{z}$, we immediately see that the magnetic permeability of a 2DHA in the metamaterial limit can be written as

$$
\mu_{e f f}=S^{2}=\frac{8 a_{x} a_{y}}{d^{2} \pi^{2}} .
$$

These two analytical expressions for $\epsilon_{\text {eff }}$ and $\mu_{\text {eff }}$ were first derived by Pendry et al. ${ }^{26}$ when analyzing the surface EM modes supported by a semi-infinite holey PEC film. The fact that the same expressions are obtained for a holey PEC film of finite thickness gives addition support to our effective medium approach. As seen from Eqs. (15) and (16), the dielectric response of a holey metal film presents a Drude-type behavior in which the plasma frequency of the electron gas is replaced by the cutoff frequency of the hole waveguide whereas the magnetic permeability is just a constant value. Therefore, Eq. (15) implies that the cutoff wavelength marks the separation between positive and negative values of $\epsilon_{e f f}$. This quasianalytical finding explains nicely the behavior of $\epsilon_{\text {eff }}$ observed in Fig. 2(b).

Due to the finite negative dielectric response of a holey PEC film, this structure supports the propagation of surface EM modes whose characteristics mimic those of SPPs, as shown next. By defining $X \equiv-Y|S|^{2} / Y_{0}$, the condition for zeros in the denominator of Eq. (13), that marks the spectral locations of the surface EM modes supported by the structure, can be expressed as

$$
\frac{1-X}{1+X}= \pm e^{-\left|q_{z}\right| h} .
$$

The condition for SPPs in a real metal film of thickness $h$ reads exactly as Eq. (17), where $X$ must be replaced by $\left(k_{z} / \epsilon\right)_{\text {metal }} /\left(k_{z} / \epsilon\right)_{\text {air }}$ and $q_{z}$ by the corresponding $k_{z}$ inside the metal. Due to their mimicking characteristics, the surface EM modes supported by a perforated PEC film are usually called spoof SPPs.

\section{DOUBLE-FISHNET STRUCTURE AND NEGATIVE-INDEX BEHAVIOR}

The rest of the paper is devoted to analyze the transmission properties and effective optical parameters of the DF structure. In the first part, and in order to simplify the discussion, we consider the case in which the dielectric film between the metal films is air $\left(\epsilon_{d}=1\right)$. Figure 3(a) renders the normal-incidence spectra for PEC and silver films. When comparing them to the spectra found for a 2DHA (see Fig. 2 ), a new resonant feature emerges in the transmission spectrum and is composed by a dip and a peak. The transmission dip appears at $\lambda_{d}^{\text {pec }}=1065 \mathrm{~nm}$ for a PEC-DF and at $\lambda_{d}^{\mathrm{Ag}}$ $=1580 \mathrm{~nm}$ for a Ag-DF. It is also important to realize that the other transmission peaks previously found for a $2 \mathrm{DHA}$ 

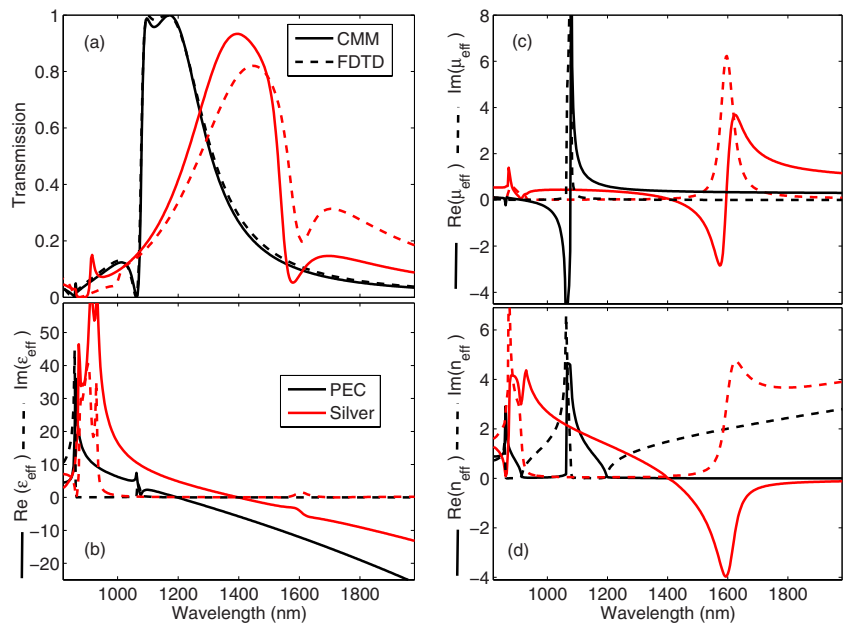

FIG. 3. (Color online) (a) Normal-incidence transmission versus wavelength for a DF in which $h_{m}=30 \mathrm{~nm}, h_{d}=50 \mathrm{~nm}$, and $\epsilon_{d}=1$. Black line: perfect electric conductor. Red line: Ag. (b) Corresponding real (solid lines) and imaginary (dashed lines) of the effective permittivity $\epsilon_{e f f}$ versus wavelength. (c) Real and imaginary part of effective magnetic permeability $\mu_{\text {eff }}$ and (d) refractive index, $n_{\text {eff }}$.

are still present in the DF spectra. As shown in Fig. 3(b), $\epsilon_{e f f}$ for the DF structures closely resembles to that found in 2DHA structures. Although a modulation of $\epsilon_{\text {eff }}$ related the new resonance is visible, the cutoff wavelength still controls the position in which $\operatorname{Re}\left(\epsilon_{e f f}\right)$ changes its sign. ${ }^{18}$

Panels (c) and (d) of Fig. 3 render the effective magnetic permeability, $\mu_{\text {eff }}$, and the effective refractive index, $n_{\text {eff }}$, respectively. Just at the spectral location of the new resonant feature, $\mu_{\text {eff }}$ exhibits negative values both for a PEC-DF and for a Ag-DF. Although in the PEC case $\mu_{\text {eff }}$ is negative, $\epsilon_{\text {eff }}$ is positive in the resonant region, leading to positive values for $\operatorname{Re}\left(n_{e f f}\right)$. On the contrary, for the Ag case, $\mu_{\text {eff }}$ exhibits negative values in the region where $\epsilon_{\text {eff }}$ is also negative. As expected, $\operatorname{Re}\left(n_{e f f}\right)$ is negative in the resonant region.

The physical origin of the magnetic resonance leading to negative values for $\mu_{\text {eff }}$ emerges clearly when looking at the electric and magnetic field patterns associated with the resonance wavelength. At the resonance wavelength for PEC, $\lambda_{d}^{p e c}=1065 \mathrm{~nm}$, the $E$-field pattern is mainly concentrated in the gap region between the metal films. In Fig. 4(a), we plot the $z$ component of the electric field, $E_{z}$, evaluated in the $x y$ middle plane between the two PEC films. The distribution has a standing wave character in the $x$ direction and the intensity is maximum along the ridges of the holes. This distribution suggests that two surface EM modes, sustained by the two metal films separated by the dielectric gap, counter propagate in the $x$ direction and are involved in the formation of the magnetic resonance. Between two adjacent holes, $E_{z}$ has opposite signs. This distribution create a displacement current that resembles that created by a magnetic dipole in the $y$ direction. The presence of this dipole modified strongly the magnetic field between the two metallic slabs as shown in Fig. 4(b).

In the dielectric region between two (real) metal slabs, gap plasmon modes created by the hybridization between the SPPs of the two interfaces can be supported. ${ }^{30}$ In the PEC
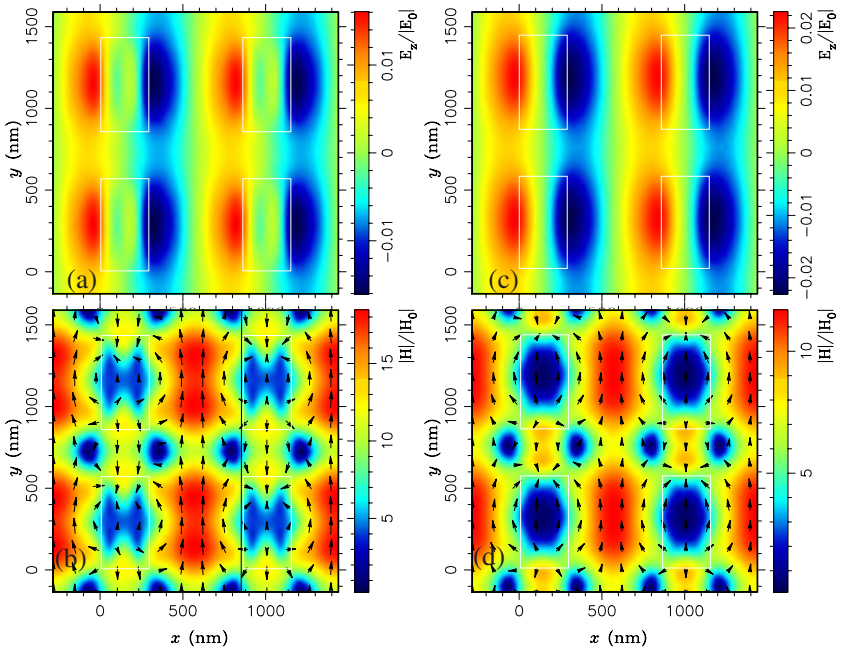

FIG. 4. (Color online) Electric field component $E_{z}$ [panels (a) and (c)] and magnetic field [panels (b) and (d)] patterns evaluated at the resonant wavelength for the DF structure. Left pictures are obtained for the PEC-DF case at $\lambda_{d}^{p e c}=1065 \mathrm{~nm}$. Results obtained at $\lambda_{d}^{\mathrm{Ag}}=1650 \mathrm{~nm}$ for Ag-DF correspond to the right pictures. The displayed field are calculated at a $x-y$ plane located in the middle of the two perforated metal films.

case, the two surface EM modes that generate the gap modes are spoof SPP modes and, therefore, the confined modes supported by a dielectric slab sandwiched between the two perforated PEC films have a geometrical origin and could be termed as gap spoof SPPs. In order to give additional support to this hypothesis, here we present some analytical work based on the simplified system of Eq. (11). The condition for zeros of the denominator reads in this case: $[(Q+\Sigma)(G-\Sigma)$ $\left.+\left(G^{V}\right)^{2}\right]^{2}-P^{2}(G-\Sigma)^{2}=0$. By using the expressions of the magnitudes involved in the PEC limit $\left(Z_{s}=0\right)$, this condition transforms into

$$
\left[\frac{1-X}{1+X}\right]^{4}-H_{1}\left[\frac{1-X}{1+X}\right]^{2} e^{-4\left|q_{z}\right| h_{m}}=0
$$

with $H_{1}=2 e^{-2\left|q_{z}\right| h_{m}}+e^{2 k_{z} h_{d}}\left(1-e^{-2 i\left|q_{z}\right| h_{m}}\right)^{2}$. This expression is exactly the same as the one derived for gap SPP modes supported a dielectric slab of thickness $h_{d}$ sandwiched between two metal films of thickness $h_{m} \cdot{ }^{30}$ As in the 2DHA case, when establishing the analogy, $X$ must be replaced by $\left(k_{z} / \epsilon\right)_{\text {metal }} /\left(k_{z} / \epsilon\right)_{a i r}$ and $q_{z}$ by the corresponding $k_{z}$ inside the metal.

In panels (c) and (d) of Fig. 4 we render the $z$ component of the electric field and the amplitude of the magnetic field pattern evaluated for Ag-DF at the resonant wavelength $\lambda_{d}^{\mathrm{Ag}}$. These distributions neatly show how the physical origin of the magnetic resonance in real metal films is very similar to that previously discussed for a PEC-DF. In the real metal case, the dielectric gap SPP modes hybridize strongly with the spoof gap SPP modes but the physical picture is maintained when going from the PEC limit to the case of a real metal at optical frequencies. It is worth noticing that by coupling the gap SPP mode to the different diffraction orders, 


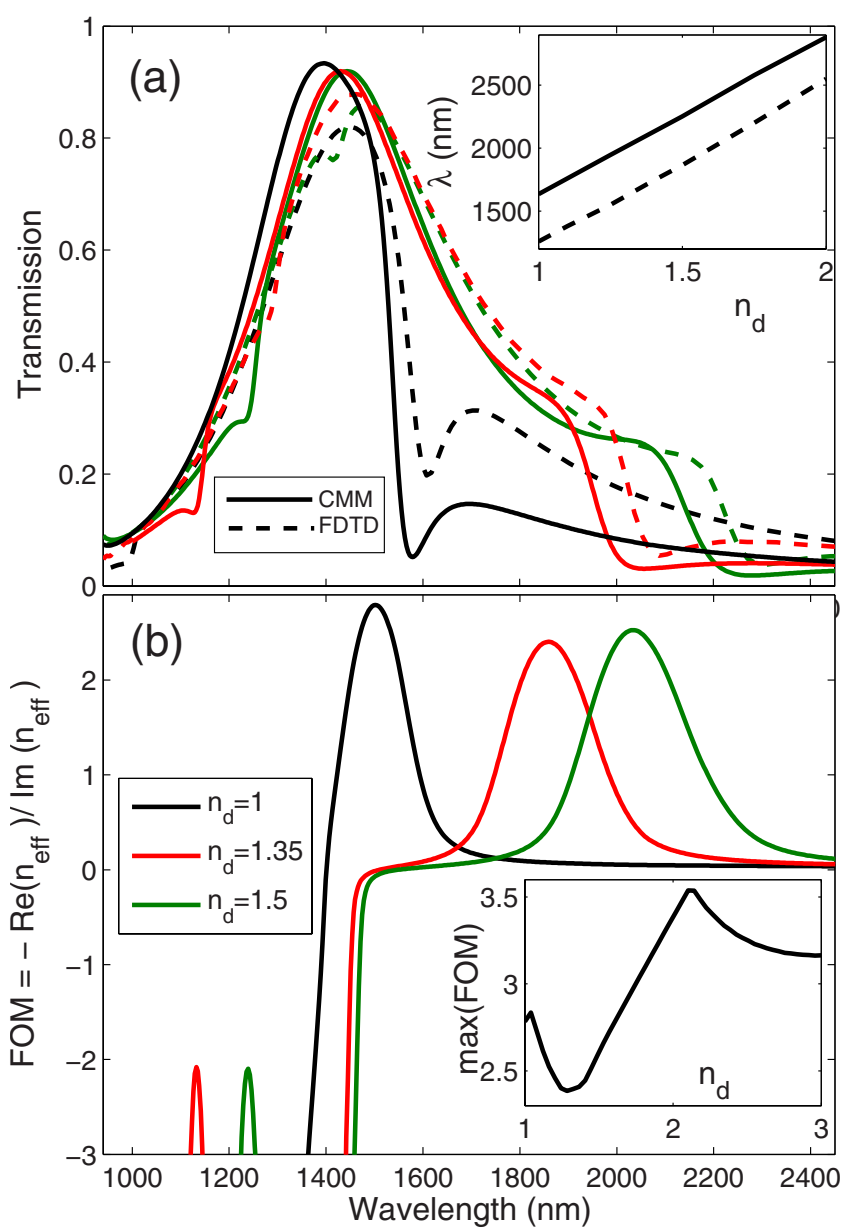

FIG. 5. (Color online) (a) Normal-incident transmission spectra for silver films. The refractive index of dielectric spacer is $n_{d}=1$ (black curves), $n_{d}=1.35$ (red curves), and $n_{d}=1.5$ (green curves). Solid line: CMM calculations. FDTD simulations without (dashed line) holes inside the dielectric layer. In the inset, the spectral location of the gap SPP mode with (solid line) and without (dashed line) holes in the metal is displayed as a function of the gap refractive index. (b) FOM versus the incident wavelength and $n_{d}=\sqrt{\epsilon_{d}}$. The inset shows the maximum of FOM versus $n_{d}$. The geometrical parameters are: thickness of the metal films, $h_{m}=30 \mathrm{~nm}$, thickness of the dielectric layer, $h_{d}=50 \mathrm{~nm}$, period of the hole array, $d$ $=860 \mathrm{~nm}$, and sides of the holes, $a_{x}=295 \mathrm{~nm}$ and $a_{y}=595 \mathrm{~nm}$. These parameters correspond to those of the experiments reported in Ref. 17.

the corresponding magnetic resonance could be spectrally tuned. ${ }^{31}$

As gap SPP modes are involved in the negative magnetic response of a DF structure, it is expected that this resonant behavior will be very sensitive to the refractive index of the dielectric slab, $n_{d}$. First of all, we note that in the experimental samples the dielectric layer is also perforated by holes. In Fig. 5(a) we validate our approach of a continuous dielectric layer by comparing the transmission spectra obtained with CMM (solid lines) with FDTD calculations for perforated dielectric films (dashed lines). Three cases are analyzed corresponding to $n_{d}=1, n_{d}=1.35$, and $n_{d}=1.5$. The similarities between the curves (there is only a small shift between the two cases, the resonant features in the nonperforated case

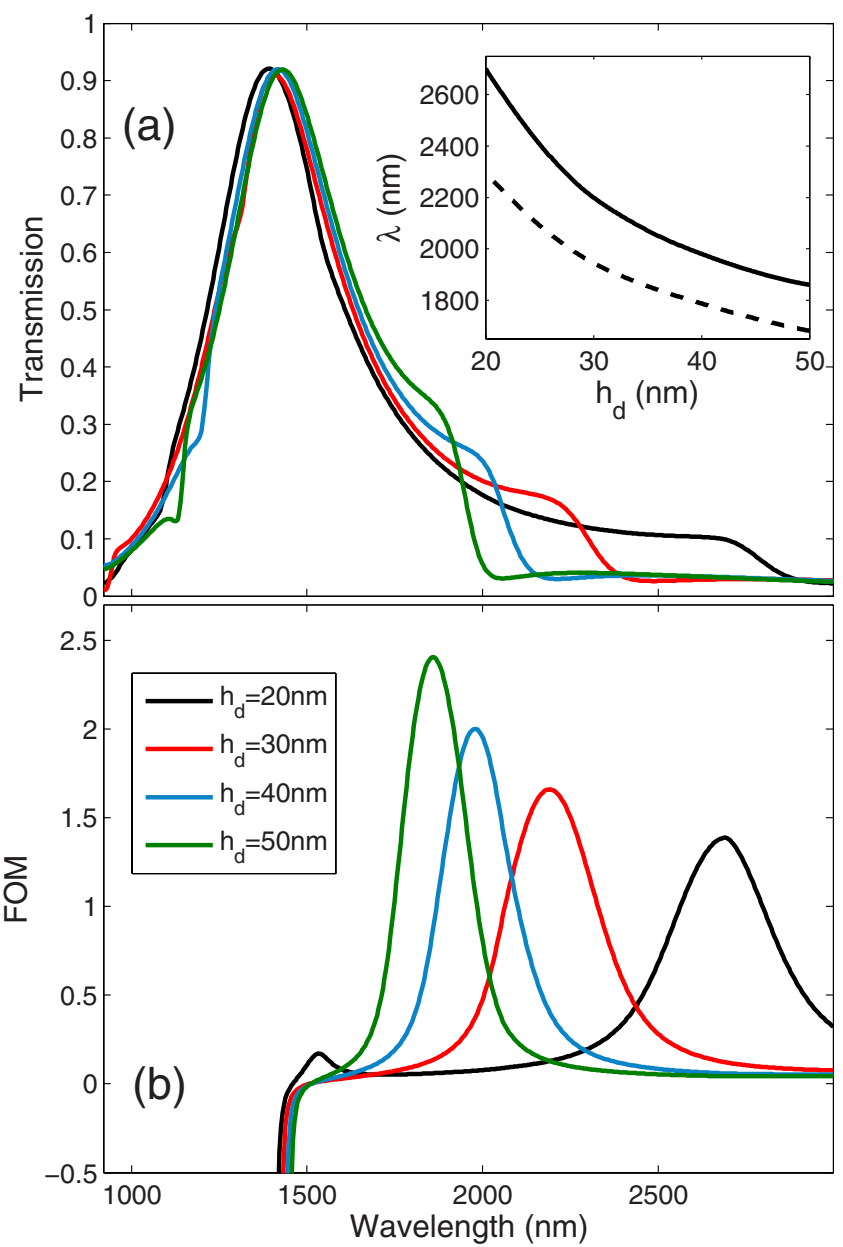

FIG. 6. (Color online) (a) Transmission spectra for normalincidence radiation as a function of the dielectric gap thickness, $h_{d}$ with $n_{d}=1.35$. In the inset, the evolution of the spectral location of the gap SPP mode for perforated Ag films (full line) and Ag films without holes (dashed line) with $h_{d}$ is displayed. (b) FOM versus wavelength for several AG-DFs of different thicknesses.

appear at slightly shorter wavelengths) demonstrate the validity of our approach.

The sensitivity of the spectral location of the magnetic resonance with $n_{d}$ is clearly visible in Fig. 5(a). Notice that, as a difference, the cutoff resonance leading to the main peak in the spectrum is quite insensitive to $n_{d}$. The inset of Fig. 5(a) further clarifies the origin of the shift to longer wavelengths of the magnetic resonance when $n_{d}$ is increased. It shows how the spectral location of the gap SPP mode excited in a dielectric slab of thickness $h_{d}=50 \mathrm{~nm}$ sandwiched between two Ag films of thickness $h_{m}=30 \mathrm{~nm}$ and evaluated at a parallel momentum $k_{x}=2 \pi / d$ changes with $n_{d}$. Two different cases are rendered: a nonperforated Ag film (dashed line) and perforated one (full line). There is an almost perfect correspondence between the spectral locations of the gap SPP modes for perforated Ag films with those of the transmission dips associated with negative $\mu_{\text {eff }}$. This result gives additional support to our finding that the negative magnetic response in DF structures is linked to the excitation of gap SPP modes. 

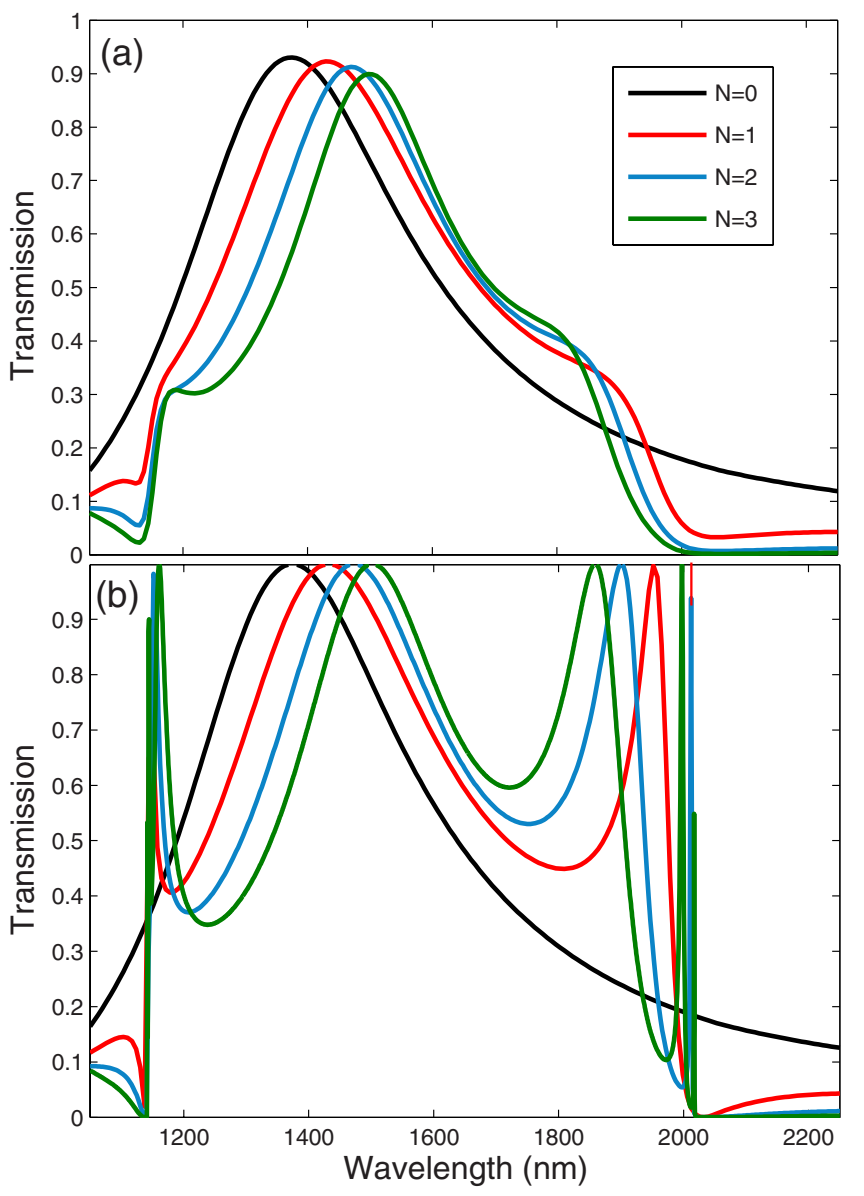

FIG. 7. (Color online) Transmission spectra through a 2DHA structure $(N=0)$ and multilayered DF structures containing different number of dielectric layers $(N=1,2,3)$ calculated with our coupledmode method. The geometrical parameters are the same as in previous figures and the dielectric film(s) are made of $\mathrm{MgF}_{2}$ with $n_{d}$ $=1.35$. In panel (a), the dielectric function is that of silver whereas in (b) absorption of silver is neglected $\{\Im[\epsilon(\lambda)]=0\}$.

In Fig. 5(b) we plot the evolution of the figure of merit (FOM) with $n_{d}$. The FOM in this context is usually defined by the following relation

$$
\mathrm{FOM}=-\frac{\mathfrak{R}\left(n_{e f f}\right)}{\mathfrak{I}\left(n_{\text {eff }}\right)} .
$$

As expected, FOM is also sensitive to $n_{d}$. Interestingly, the inset in Fig. 5(b) demonstrates that this FOM can be optimized by an appropriate election of the dielectric medium located between the two holey metal films. For this set of geometrical parameters, the optimum $n_{d}$ seems to be on the order of 2.

Another parameter that controls the spectral location of the gap SPP modes is the thickness of the dielectric layer. In Fig. 6 we render the transmission spectra and the corresponding FOM for several dielectric thicknesses, $h_{d}$. For these simulations the dielectric medium is chosen to be $\mathrm{MgF}_{2}$ $\left(n_{d}=1.35\right)$, as in the experiments reported in Ref. 17. The spectral location of the transmission dip moves to shorter wavelengths when $h_{d}$ is decreased and, importantly, FOM

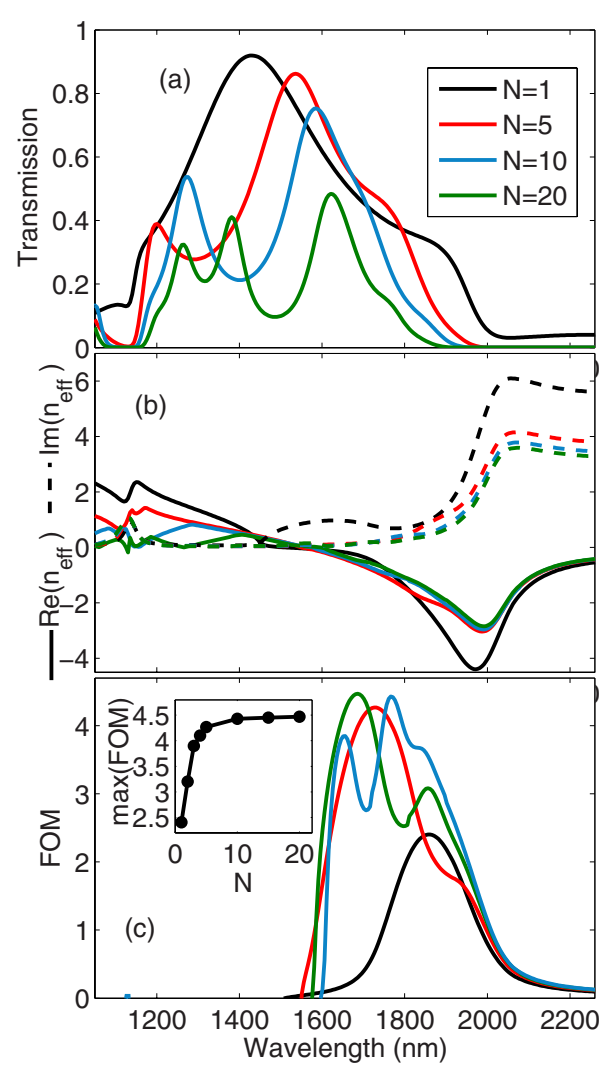

FIG. 8. (Color online) (a) Transmission spectra through multilayered DF structures containing different number of dielectric layers $(N=1,5,10,20)$ calculated with our coupled-mode method. The geometrical parameters are the same as in previous figures. (b) The retrieved effective refractive index (real and imaginary parts) for the cases analyzed in panel (a). (c) The corresponding FOM versus wavelength. Inset renders the maximum of FOM as a function of the number of dielectric layers.

increases for thick dielectric slabs. Again, the evolution of the spectral location of the magnetic resonance with $h_{d}$ can be explained by looking at the behavior of the gap SPP modes [see inset of Fig. 6(a)]. When $h_{d}$ is increased, the resonance wavelength shifts to shorter wavelengths as the EM coupling between the two SPP modes of the two metal films forming the gap SPP mode becomes smaller.

\section{MULTILAYERED DF STRUCTURES}

In this section we present how the transmission spectrum evolves from the case of a DF structure to CDF structure composed by a discrete number of DFs. Figure 7(a) shows the transmission spectra calculated with the coupled-mode method. $N$ refers to the number of dielectric spacers (which means $N+1$ holey metal films). The geometrical parameters are the same as in the previous section and correspond to those reported experimentally in Ref. 17.

In order to clearly identify the resonances, it is convenient to switch off the absorption inside the metal layers in our numerical method (CMM). This is done in Fig. 7(b) where we render the same cases analyzed in Fig. 7(a) but considering $\Im[\epsilon(\lambda)]=0$. Within this approach, all the light is being 
either transmitted or reflected. As clearly seen in this figure, the inclusion of more and more gap layers results in the appearance of additional resonant features in the transmission spectra. ${ }^{14,15}$ The number of these new features exactly coincides with twice the number of dielectric layers, $N$. We can understand this behavior by realizing that the addition of a dielectric layer introduces two new gap SPP modes into the picture. These gap SPP modes localized in the different dielectric layers are coupled evanescently via the holes of the metal films. We also note that the linewidths of the transmission peaks are strongly reduced as $N$ is increased. This behavior explains why the presence of absorption within the metal layers strongly limits the heights of the transmission peaks in multilayered DF structures.

Finally we analyze how the negative refractive-index response and the corresponding FOM evolves when the number of dielectric layers is largely increased. In Fig. 8(a), we show the transmission spectra for CDF structures containing up to 20 dielectric layers. This number of dielectric spacers is similar to the bulk CDF recently analyzed by Valentine et $a l{ }^{17}$ As in the previous theoretical ${ }^{15}$ and experimental works, ${ }^{17}$ FOM increases as more dielectric layers are incorporated into the structure. However, there is a kind of saturation [see inset of Fig. 8(c)] that, for this particular set of geometrical parameters seems to be between 5 and 10 .

\section{CONCLUSIONS}

We have presented an unified quasianalytical model for light transmission through holey metal films and doublefishnet structures. We have revisited in detail the physical mechanisms leading to enhanced transmission in 2DHAs and negative refractive-index response in DF structures. By looking at the spectral locations of the peaks as a function of dielectric layer properties (dielectric constant and thickness), we have provided further evidence that the resonant magnetic response that yields negative values for the effective magnetic permeability in DF structures is related to the excitation of gap SPP modes propagating along the dielectric gap. We have also studied the evolution of the negativeindex behavior with the number of DF unit cells, finding that the bulk value for the effective refractive index is reached for a very small number of DF layers.

\section{ACKNOWLEDGMENTS}

Financial support by the EU under Contract No. ISTFP6034506 and Spanish Ministry of Science under Projects No. MAT2008-06609-C02 and No. CSD2007-046-NanoLight.es is gratefully acknowledged.
${ }^{1}$ T. W. Ebbesen, H. J. Lezec, H. F. Ghaemi, T. Thio, and P. A. Wolff, Nature (London) 391, 667 (1998).

${ }^{2}$ C. Genet and T. W. Ebbesen, Nature (London) 445, 39 (2007).

${ }^{3}$ F. J. Garcia-Vidal, L. Martin-Moreno, T. W. Ebbesen, and L. Kuipers, Rev. Mod. Phys. (to be published).

${ }^{4}$ H. F. Ghaemi, T. Thio, D. E. Grupp, T. W. Ebbesen, and H. J. Lezec, Phys. Rev. B 58, 6779 (1998).

${ }^{5}$ L. Martín-Moreno, F. J. García-Vidal, H. J. Lezec, K. M. Pellerin, T. Thio, J. B. Pendry, and T. W. Ebbesen, Phys. Rev. Lett. 86, 1114 (2001); L. Martín-Moreno and F. J. García-Vidal, Opt. Express 12, 3619 (2004).

${ }^{6}$ S. Zhang, W. Fan, N. C. Panoiu, K. J. Malloy, R. M. Osgood, and S. R. J. Brueck, Phys. Rev. Lett. 95, 137404 (2005).

${ }^{7}$ S. Zhang, W. Fan, K. J. Malloy, S. R. J. Brueck, N. C. Panoiu, and R. M. Osgood, Opt. Express 13, 4922 (2005).

${ }^{8}$ C. M. Soukoulis, S. Linden, and M. Wegener, Science 315, 47 (2007).

${ }^{9}$ G. Dolling, C. Enkrich, M. Wegener, C. M. Soukoulis, and S. Linden, Opt. Lett. 31, 1800 (2006).

${ }^{10}$ G. Dolling, C. Enkrich, M. Wegener, C. M. Soukoulis, and S. Linden, Science 312, 892 (2006).

${ }^{11}$ M. Beruete, M. Sorolla, I. Campillo, J. S. Dolado, L. MartínMoreno, J. Bravo-Abad, and F. J. García-Vidal, Opt. Lett. 29, 2500 (2004).

${ }^{12}$ M. Beruete, M. Sorolla, and I. Campillo, Opt. Express 14, 5445 (2006).

${ }^{13}$ M. Kafesaki, I. Tsiapa, N. Katsarakis, Th. Koschny, C. M. Soukoulis, and E. N. Economou, Phys. Rev. B 75, 235114 (2007).

${ }^{14}$ T. Li, H. Liu, F. M. Wang, Z. G. Dong, S. N. Zhu, and X. Zhang, Opt. Express 14, 11155 (2006).

${ }^{15}$ S. Zhang, W. Fan, N. C. Panoui, K. J. Malloy, R. M. Osgood, and S. R. Brueck, Opt. Express 14, 6778 (2006).
${ }^{16}$ G. Dolling, M. Wegener, and S. Linden, Opt. Lett. 32, 551 (2007).

${ }^{17}$ J. Valentine, S. Zhang, T. Zentgraf, E. Ulin-Avila, D. A. Genov, G. Bartal, and X. Zhang, Nature (London) 455, 376 (2008).

${ }^{18}$ A. Mary, Sergio G. Rodrigo, F. J. Garcia-Vidal, and L. MartinMoreno, Phys. Rev. Lett. 101, 103902 (2008).

${ }^{19}$ M. Beruete, M. Navarro-Cía, M. Sorolla, and I. Campillo, Phys. Rev. B 79, 195107 (2009).

${ }^{20}$ A. Mary, Sergio G. Rodrigo, L. Martin-Moreno, and F. J. GarciaVidal, J. Phys. C 20, 304215 (2008).

${ }^{21}$ J. Bravo-Abad, F. J. García-Vidal, and L. Martín-Moreno, Phys. Rev. Lett. 93, 227401 (2004).

${ }^{22}$ F. de León-Pérez, G. Brucoli, F. J. García-Vidal, and L. MartínMoreno, New J. Phys. 10, 105017 (2008).

${ }^{23}$ D. R. Smith, S. Schultz, P. Markos, and C. M. Soukoulis, Phys. Rev. B 65, 195104 (2002).

${ }^{24}$ J. B. Pendry, A. J. Holden, D. J. Robbins, and W. J. Stewart, IEEE Trans. Microwave Theory Tech. 47, 2075 (1999).

${ }^{25}$ A. Mary, S. G. Rodrigo, L. Martín-Moreno, and F. J. GarcíaVidal, Phys. Rev. B 76, 195414 (2007).

${ }^{26}$ J. B. Pendry, L. Martín-Moreno, and F. J. Garcia-Vidal, Science 305, 847 (2004); F. J. Garcia-Vidal, L. Martín-Moreno, and J. B. Pendry, J. Opt. A, Pure Appl. Opt. 7, S97 (2005).

${ }^{27}$ F. J. García-Vidal, Esteban Moreno, J. A. Porto, and L. MartínMoreno, Phys. Rev. Lett. 95, 103901 (2005).

${ }^{28}$ R. Gordon and A. G. Brolo, Opt. Express 13, 1933 (2005).

${ }^{29}$ F. J. García-Vidal, L. Martín-Moreno, Esteban Moreno, L. K. S. Kumar, and R. Gordon, Phys. Rev. B 74, 153411 (2006).

${ }^{30}$ E. N. Economou, Phys. Rev. 182, 539 (1969).

${ }^{31}$ C. García-Meca, R. Ortuño, F. J. Rodríguez-Fortuño, J. Martí, and A. Martínez, Opt. Lett. 34, 1603 (2009). 